\title{
Under cover
}

\author{
Stephen Hancocks OBE \\ Editor-in-Chief
}

The BDJ Upfront section includes editorials, letters, news, book reviews and interviews. Please direct your correspondence to the News Editor,

Kate Quinlan at BDJNews@nature.com. Press releases or articles may be edited, and should include a colour photograph if possible.

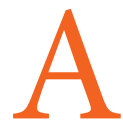

lthough they come under fire from time to time from those who have

different tastes, ideas of how a 'serious' dental journal should look or alternative views in general, we spend a lot of time and thought on our cover series. We believe that the images we select help to create the unique, and well respected publication that this journal has grown to become nationally and internationally. In this we are enormously grateful for the skills of our Art Editor, Melissa Cassem.

The current series, while visually impactful, is also directly related to dentistry in that the images are cropped from original oral hygiene education (for so it was termed in those days) materials in the collection of the BDA Museum. We hope that readers will enjoy them as well as ponder on the thinking behind some of the messages given. In some cases artistic licence has been stretched to make them visually arresting and memorable. In the realm of advertising, complex messages have to be delivered with extreme brevity but in doing so can unwittingly preserve a snapshot of the culture and society in which they are created.

On the cover of this issue we reproduce part of a public information sheet from the late 1940s which was one of a series produced by the Ministry of Health. 'Unsound teeth undermine general health. Poisons from bad teeth cause abscesses: the discharge is swallowed and makes us tired and listless. It poisons the blood stream and may cause serious injury to our health' declares the doomsday style text. Both the man and woman pictured look depressed and despondent apparently from the effects of infected teeth. Two things are immediately striking about these time-rooted images. Firstly, the depiction of the man at rest in an armchair in suit and tie, perhaps home from work, while the woman is shown as cleaning, perhaps her own home or maybe that of the man in the other picture. I think we would brand these today as stereotypical. Secondly, the message, which we might nowadays scoff at as being at best naïve and at worst bordering on nonsense. Or is it?

While the wording and any evidence-base may be distinctly questionable the sentiment is not so far removed from our current philosophy that oral health and general health are inextricably linked. Indeed we only have to look to four 2017 'evidence summaries' published in the $B D J$ to find the following conclusions:

- 'There is high quality evidence to support an association between cardiovascular disease and oral health' ${ }^{1}$

- 'The cumulative evidence of this review suggests an association between oral and pulmonary disease, specifically COPD [chronic obstructive pulmonary disease] and pneumonia, and incidence of the latter can be reduced by oral hygiene measures ${ }^{2}$ commissioned an animated cartoon version of teeth explaining the associations between oral health and the general health conditions researched. This is available to view at 'Oral health is not just about the mouth' on the $B D J$ YouTube channel (http://bit.ly/oralhealthvid).

Our post-second world war doyens of domestic harmony slumped in their chairs on this issue's cover could never have foreseen the development of understanding in the intervening seventy or so years. Yet, somewhat ironically, their war-rationed diets were arguably more healthy then than our opulent, obesity-causing equivalents today. Perhaps there wasn't such an obvious link at that time between these diseases because the incidence of cardiac conditions and diabetes were lower.

This under-cover, cover artwork has

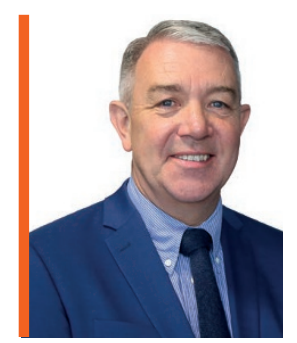

\section{'Poisons from bad teeth cause abscesses: the discharge is swallowed and makes us tired and listless...'}

- 'Current evidence, of mixed quality, suggests a number of associations between oral diseases and diabetes mellitus (diabetes) ${ }^{3}$

- 'Suboptimal oral health (gingivitis, dental caries, tooth loss, edentulousness) appears to be associated with increased risk of developing cognitive impairment and dementia.'

Not quite the 'poison' of the Ministry's dire warning but given our knowledge at the time, and more pertinently the knowledge of the general population - for example, who knew then that plaque caused gum disease? - it is not a bad approximation of our 'modern' mantra. Taking the progression one step further, in order to emphasise the conclusions of the evidence summaries, the $B D J$ thrown up some interesting points of discussion on the value of looking back as well as forwards. Whatever the medium, the message has stayed remarkably consistent; if you maintain good oral health then it can do no harm at all to your general health. What will that be like seventy more years on?

DOI: 10.1038/sj.bdj.2018.46

1. Dietrich T, Webb I, Stenhouse $L$ et al. Evidence summary: the relationship between oral and cardiovascular disease. Br Dent J 2017; 222: 381-385.

2. Mange D, Walshaw M, Fitzgerald et al. Evidence summary: the relationship between oral health and pulmonary disease. Br Dent J 2017; 222: 527-533.

3. D'Aiuto F, Gable D, Syed Z et al. Evidence summary: The relationship between oral diseases and diabetes. Br Dent J 2017; 222: 944-948.

4. Daly B, Thompsell A, Sharpling J et al. Evidence summary: the relationship between oral health and dementia. Br Dent J 2017; 223: 846-853. 